\title{
Atelier « Science and values in radiological protection »
}

\author{
L. LEBARON-JACOBS ${ }^{1}$, E. GAILLARD-LECANU ${ }^{2}$
}

(Manuscrit reçu le 14 février 2008, accepté le 17 mars 2008)

RÉSUMÉ L'AEN (Agence pour l'énergie nucléaire) a organisé un atelier «Sciences and values in radiological protection » afin d'identifier les voies possibles d'intégration en radioprotection de certains résultats du domaine de la recherche sur les effets biologiques et sur la santé des rayonnements ionisants. Trois thèmes d'actualité ont été développés : les effets non ciblés, la radiosensibilité individuelle et les maladies cardio-vasculaires. À l'issue de ces journées, des recommandations ont été émises envers les scientifiques, les radioprotectionnistes et les parties prenantes.

ABSTRACT Workshop "Sciences and values in radiological protection".

The NEA (Nuclear Energy Agency) organized a workshop entitled "Sciences and values in radiological protection" in order to identify the possible ways of integration of results, coming from scientific research on the biological and health effects of ionizing radiation, in radiological protection. Three current topics were addressed: non-targeted effects, individual radiosensitivity and cardiovascular diseases. At the end of this workshop, recommendations towards scientists, radiological protection practitioners and stakeholders were expressed.

Keywords: bystander effect / genomic instability / individual radiosensitivity / cardiovascular diseases / non-targeted effects

« The establishment of maximum permissible radiation levels is not a scientific question, but it must be based on scientific knowledge » (Sievert, 1956).

\section{Introduction}

Depuis 1958, les recommandations successives de la CIPR (Commission de protection radiologique) se sont attachées à faire évoluer le choix des limites de dose, mais aussi les règles de radioprotection (justification des expositions, optimisation, ...) en fonction des résultats scientifiques (épidémiologie, études expérimentales, études des mécanismes biologiques).

L'AEN (Agence pour l'énergie nucléaire), au travers du CRPPH (Committee on Radiation Protection and Public Health) a constaté un besoin, émanant à la fois des autorités, des parties prenantes, mais aussi des acteurs de terrain, de mieux

1 DSV/DIR/CARMIN, CEA Cadarache, 13108 Saint-Paul-Lez-Durance Cedex, France.

2 DSV/DIR/CARMIN, CEA Fontenay-aux-Roses, 92265 Fontenay-aux-Roses, France. 
identifier les interactions possibles entre les recherches en cours sur les effets biologiques et sur la santé des rayonnements ionisants et le développement d'une politique de radioprotection.

Des résultats récents sur les atteintes cellulaires indirectes après irradiation (effet bystander, instabilité génétique, ...) et l'augmentation possible, dose dépendante, d'effets non cancérogènes (cardio-vasculaire, pulmonaire, hépatique, ...) sont en effet à l'origine de la réflexion en cours sur leurs implications potentielles en radioprotection.

En 2007, deux groupes de travail du CRPPH (EGIS : Expert group on the Implications of Radiological Protection Science ; EGCO : Expert Group on the Collective Opinion) ont publié chacun un rapport :

- le premier rassemblant les questions scientifiques d'importance qui pourraient avoir des implications potentielles en radioprotection (NEA, 2007) ;

- le second identifiant les apports possibles du système de radioprotection actuel dans l'aide à la décision de nos autorités (NEA, 2008).

Les objectifs de ce workshop étaient de réfléchir aux éventuelles pistes d'intégration des données scientifiques en radioprotection, à l'adéquation entre les questions posées en radioprotection et les réponses apportées par la recherche, tout en tenant compte des considérations sociétales (application du principe de précaution, acceptabilité du risque, ....).

Après des exposés généraux sur les thèmes abordés, les participants se sont répartis en trois groupes de travail abordant séparément les trois thèmes scientifiques qui suscitent particulièrement l'intérêt à l'heure actuelle : les effets non ciblés, la radiosensibilité individuelle et les maladies cardiovasculaires.

\section{Les effets non ciblés (ENC) (effet bystander, instabilité génétique)}

Ce groupe de travail, dont les débats ont été rapportés par Henri Métivier, président du groupe de travail de l'AEN, EGIS, a soulevé plus de questions qu'il n'en a résolues. L'exercice était difficile car la première question qui vient à l'esprit « les effets non ciblés mis en évidence in vitro, parfois à des doses faibles, conduisentils à un effet sur la santé ?»n'a pas encore trouvé sa solution dans les données scientifiques de la recherche en biologie.

En termes de conséquences pour la radioprotection, les résultats des recherches sur les effets non ciblés sont particulièrement attendus dans le domaine des faibles doses, où l'évaluation du risque est réalisée par l'extrapolation selon une relation 
linéaire sans seuil, à faible dose, du risque observé à plus forte dose. Les recherches sur les ENC donnent des informations sur les mécanismes biologiques mis en œuvre, et apportent des indications expérimentales directes y compris dans le domaine dit « des faibles doses » (schématiquement au-dessous d'une centaine de $\mathrm{mSv}$ pour les adultes et quelques dizaines de $\mathrm{mSv}$ pour les jeunes enfants). Il reste à savoir si une meilleure compréhension des mécanismes biologiques sera suffisante pour réduire les incertitudes actuelles sur l'évaluation des risques à faibles doses.

Le choix de la relation dose-effet, notamment pour l'évaluation des risques des faibles doses, doit s'appuyer sur les connaissances scientifiques et les données épidémiologiques. Pour des doses supérieures à $200 \mathrm{mSv}$ environ, les données de l'épidémiologie permettent d'apprécier la relation dose-effet avec une certaine précision. En revanche, pour des faibles doses (inférieures à $200 \mathrm{mSv}$ ), rencontrées le plus souvent dans le domaine de la radioprotection, les études épidémiologiques ne permettent ni d'affirmer l'existence d'un excès de cancer, ni d'exclure son éventualité. L'utilisation d'une relation linéaire sans seuil (RLSS) pour estimer le risque des faibles doses est rendue possible grâce à l'extrapolation de la relation, le plus souvent linéaire, qui est observée entre la dose et la probabilité d'occurrence de l'effet cancérogène pour les doses supérieures à $200 \mathrm{mSv}$. Ces notions existent depuis longtemps; en revanche, il est peu vraisemblable que de nouvelles études parviennent, dans un avenir proche, à estimer ces risques éventuels et encore moins à les exclure.

Le groupe de travail a constaté que la relation dose-effet, pour les ENC, est parfois très différente d'une relation linéaire sans seuil (courbe saturante avec plateau par exemple). Quelles peuvent en être les conséquences en radioprotection? La relation linéaire sans seuil reste-t-elle applicable à faible dose sachant que les ENC sont des effets biologiques et non des effets sur la santé ? Les mécanismes biologiques à l'origine des ENC sont-ils les mêmes quelques soient la dose et le débit de dose?

Il est clairement établi que le cancer est la conséquence directe et aléatoire d'une lésion de l'ADN dans une cellule capable de se diviser. De nombreuses études ont montré que, au cours du processus de cancérogenèse, l'environnement de la cellule, ses interactions avec les cellules saines ainsi que les mécanismes de régulation de la prolifération liés à la structure tissulaire jouent un rôle capital. Le développement d'un cancer est par conséquent une réponse de l'organe, même si son origine est clonale.

Enfin, des questions de biologie plus fondamentale sont aussi posées au travers des recherches menées sur les faibles doses de rayonnements ionisants. L'instabilité génomique et l'effet bystander sont des phénomènes présents en 
permanence au niveau cellulaire. On sait que l'instabilité génomique peut être induite par de nombreux agents. L'effet bystander est-il spécifique des rayonnements ionisants? Existe-t-il un lien entre ces ENC et l'apparition de mutations radio-induites précoces à faibles doses ?

En conclusion, il est proposé :

1. d'associer les recherches dans le domaine des ENC à celles plus générales sur le cancer, en s'attachant à caractériser une éventuelle relation entre les ENC et les autres maladies (potentiellement radio-induites) ;

2. de développer des recherches sur l'étude d'un lien entre effets biologiques et effets sur la santé ;

3. une mise en garde particulière des scientifiques à bien valider leurs résultats avant leur application possible en radioprotection, et à ne pas confondre recherche en radioprotection et recherche dans le domaine des effets biologiques des rayonnements ionisants.

\section{La radiosensibilité individuelle}

Les travaux de ce second groupe de travail ont été rapportés par Ted Lazo (AEN). D'un point de vue de santé publique, l'hypersensibilité est un enjeu très important pour la radiothérapie. Elle concerne environ $5 \%$ des patients traités. Il est urgent, afin d'améliorer les traitements de radiothérapie, de pouvoir identifier ces patients («marqueurs biologiques de l'hypersensibilité »), ainsi que les patients hyposensibles, pour lesquels les traitements pourraient aussi être améliorés. L'hyper/hyposensibilité est une préoccupation de cliniciens. On peut espérer disposer de tests fiables dans quelques années (West et al., 2007).

Dans le domaine de la recherche, les études sur l'hypersensibilité à forte dose peuvent aussi apporter des informations sur ce même phénomène à plus faible dose. L'un des enjeux est de définir les critères biologiques représentatifs de l'hypersensibilité : suivant que l'on recherche une hypersensibilité précoce (par exemple se traduisant par des réactions tissulaires précoces) ou une hypersensibilité tardive (par exemple se traduisant par un risque augmenté de cancer), les critères biologiques peuvent être différents.

Afin d'être en mesure d'évaluer les conséquences de ce phénomène pour la radioprotection, les recherches doivent se poursuivre pour quantifier l'amplitude de l'hypersensibilité, la proportion de la population concernée, les conséquences éventuelles en termes d'effets stochastiques et/ou déterministes, selon le niveau de dose et celui de l'hypersensibilité. Les moyens utilisables (études épidémiologiques, épidémiologie moléculaire, études in vitro et in vivo) les plus pertinents restent sujets à discussion. Les propriétés de l'hypersensibilité 
(pérennité de la «signature », évolution dans le temps, influence des facteurs épigénétiques, des conditions de vie, ...) doivent aussi être caractérisées.

En effet, pour la radioprotection, l'incidence va dépendre en particulier de l'amplitude de l'hypersensibilité (facteur 2, facteur 10 ?) et de la population concernée, propriétés qui restent à caractériser. Les éléments de réponse issus de l'observation des patients soignés par radiothérapie ne sont pas nécessairement représentatifs des caractéristiques de la radiosensibilité individuelle de la population générale.

Actuellement les catégories de personnes ayant une hypersensibilité «modeste » mais connue (jeunes enfants, femmes, femmes enceintes) ne sont pas prises en compte dans les réglementations de radioprotection (une seule limite moyenne des valeurs et non valeur la plus faible). Si un tel phénomène devait être considéré dans les règles de radioprotection, quels seraient alors les moyens les plus pertinents? Instauration de limites particulières? Alignement sur les limites les plus basses ? Ces questions sont essentielles dans le cas de l'exposition à des doses faibles ou moyennes (en cas d'intervention pour sauver des vies, elles peuvent atteindre plusieurs centaines de $\mathrm{mSv}$ ).

De manière plus générale (et hors du champ de la radiothérapie), la détection $a$ priori de l'hypersensibilité, par des tests génétiques prédictifs d'un éventuel futur effet sur la santé du à cette hypersensibilité, pose des questions éthiques, qui rentrent en particulier dans le cadre de l'accord international sur la nondiscrimination par des considérations génétiques ${ }^{3}$ (UNESCO, 2003) Ces considérations éthiques portent en particulier sur la non discrimination au travail et la délivrance des aptitudes.

Si l'on sort du champ de la radiothérapie, une question de radioprotection pourrait se poser dans le cas d'examens médicaux particulièrement «dosants », suivant l'importance de l'hypersensibilité individuelle : quelles pourraient être les conséquences en termes de fréquence des examens, ce paramètre serait-il à prendre en compte dans le processus de justification?

\section{Les maladies cardio-vasculaires - CVD}

Le titre a été modifié au cours de la table ronde, le premier «maladies circulatoires » n'étant pas suffisamment précis. Le rapport fut réalisé par Miroslav Pinak (AEN).

\footnotetext{
3 International declaration on human genetic data (2003): "every effort should be made to ensure that human genetic data and human proteomic data are not used for purposes that discriminate in a way that it is intended to infringe, or has the effect of infringing human rights, fundamental freedoms or human dignity of an individual or for purposes that lead to the stigmatization of an individual, a family, a group or communities".
} 
L'exposition cardiaque et de l'appareil circulatoire à de fortes doses de rayonnements ionisants (plusieurs grays) augmente le risque de développer à long terme des maladies cardio-vasculaires (infarctus, HTA, arrêt cardiaque, accident vasculaire cérébral). De plus, le suivi des survivants d'Hiroshima-Nagasaki et de certaines populations exposées à des doses « relativement faibles » (inférieures à 2 Gy) montrerait un lien entre leur exposition et l'augmentation du risque de développer des maladies cardio-vasculaires. Pour Preston (2008), le risque relatif de présenter une telle pathologie est bien plus faible que celui de développer un cancer après exposition aux rayonnements ionisants.

1. Les études épidémiologiques chez les survivants d'Hiroshima-Nagasaki montrent une incidence significative de ces pathologies à partir de $0,5 \mathrm{~Sv}$, qui peuvent avoir un impact significatif sur la mortalité et la morbidité. C'est une question émergente en radioprotection, et qui fait l'objet d'un intérêt croissant de la part de certaines parties prenantes. Durant la discussion générale qui a suivi la présentation des conclusions, il a été souligné que si ces résultats étaient extrapolés aux doses moyennes ou faibles (par exemple dans le cas des expositions médicales), cela conduirait à une explosion du nombre de CVD potentiellement radio-induites. Pour des doses inférieures au Gy, les seuls résultats indiquant une augmentation du risque de CVD sont ceux d'Hiroshima-Nagasaki. Tous les autres résultats épidémiologiques sont négatifs. En revanche, ce phénomène a été clairement établi dans le cadre des conséquences des radiothérapies pour cancer du sein.

2. Il est nécessaire d'approfondir les données sur l'existence ou non d'un seuil et plus généralement la forme de la relation dose-réponse, afin de mieux cerner les éventuelles conséquences en termes de radioprotection.

3. Une meilleure caractérisation des CVD implique d'étudier les mécanismes biologiques et les tissus cibles. Il est également indispensable de mieux cerner l'influence de différents facteurs tels que le débit de dose et la qualité du rayonnement en cas d'exposition aiguë ou chronique, l'âge et le sexe, ainsi que les modes d'interaction avec d'autres agents.

Parallèlement, il a été souligné que les études épidémiologiques recherchant une augmentation du risque de CVD avaient toutes (sauf Hiroshima-Nagasaki) été construites pour étudier d'autres risques (cancers). En particulier, les registres des cas exposés et des témoins sont très insuffisants (en nombre et en qualité des renseignements). Des études ciblées sur ce risque ont été mises en place récemment. Il est possible que les conclusions évoluent en conséquence lorsque leurs résultats seront disponibles. 
De quelles données avons-nous besoin ?

- En radiobiologie : aux fortes doses, quelques études tendent à montrer le rôle de la cellule endothéliale dans l'inflammation radio-induite. Qu'en est-il aux faibles doses? Y a-t-il un tissu cible? Des études débutent sur le sujet (coordination par Guido Hildebrandt, Université de Leipzig, d'un sous-projet du programme Euratom et santé du 6 e PCRD) (Hildebrandt, 2006).

- En épidémiologie : des études à des doses inférieures à $0,5 \mathrm{~Sv}$ sont en cours. Une question se pose : le risque relatif dépend-il du type de pathologie cardiovasculaire?

4. En l'état actuel des choses, si l'on considérait l'estimation du risque réalisée à partir des données d'Hiroshima - Nagasaki et de la relation linéaire sans seuil, le détriment devrait augmenter de 50 à $100 \%$, conduisant à un abaissement des limites de dose de 30 à $50 \%$. Toutefois, même dans ce cas, le bénéfice en termes de santé publique restera mineur, voire indétectable, étant donné le nombre de CVD induites par d'autres facteurs que les rayonnements ionisants.

5. Dans le domaine médical, certaines expositions (par exemple en tomodensitométrie $\mathrm{X}$ ) sont au moins 100 fois supérieures à celles des travailleurs. Il est important de mieux informer les praticiens dès maintenant sur ce risque potentiel dans la mesure où le principe de limite de dose ne n'applique pas aux patients.

6. L'importance de la réflexion en cours à l'UNSCEAR sur ce thème a été soulignée. La CIPR sera attentive à l'évolution des données sur les CVD. La question de l'application du principe de précaution, dans ce contexte où aucun mécanisme biologique n'est privilégié, a aussi été abordée.

7. Que devrions-nous entreprendre au jour d'aujourd'hui ?

- un renforcement des études scientifiques sur les mécanismes biologiques et l'épidémiologie ;

- une revue critique des données de la littérature ;

- une amélioration de la prise de conscience des professionnels sur les risques potentiels notamment pour de l'exposition médicale.

\section{Conclusion}

Au cours de ce workshop, l'état des lieux des recherches fondamentales sur les effets non ciblés induits par les rayonnements ionisants, la radiosensibilité individuelle ainsi que les maladies cardiovasculaires radio-induites a été dressé. Ces recherches ont été mises en perspective dans le cadre de leurs implications 
potentielles sur l'évaluation du risque des effets sanitaires des rayonnements ionisants et de leurs conséquences sur les règles de radioprotection.

Néanmoins, à l'issue de ce workshop, on constate qu'un long chemin reste encore à parcourir avant de pouvoir intégrer certaines données scientifiques au champ de la radioprotection. D'ailleurs, les nouvelles recommandations de la CIPR 103 n'ont pas pris en compte ces questions, pourtant d'actualité, du fait de leur caractère non conclusif.

Ainsi, des recherches sont à mener notamment sur :

- les effets non ciblés dans le domaine des faibles doses, afin d'établir un lien éventuel entre les effets biologiques et sur la santé des rayonnements ionisants,

- le développement de tests génétiques prédictifs de radiosensibilité individuelle, essentiels pour la radiothérapie, et la caractérisation de l'amplitude du phénomène pour des doses hors radiothérapie,

- les mécanismes biologiques à l'origine des maladies cardio-vasculaires radioinduites, et la caractérisation de l'occurrence de ces maladies pour le domaine de doses de la radioprotection.

Des recommandations, s'adressant aux scientifiques et aux radioprotectionnistes, et identifiant les priorités de recherche, ont été formulées au sein des trois groupes de travail afin d'asseoir les bases de la radioprotection dans le futur.

Les communications qui ont été présentées lors de ce workshop sont disponibles sur le site de l'AEN http://www.nea.fr/html/rp/helsinki08/programme. html.

\section{RÉFÉRENCES}

Hildebrandt G. (2006) Non-cancer diseases, from NOTE: Non-targeted effects of ionizing radiation, Integrated project 2006-2010, supported by 6th Framework programme.

NEA (2007) Scientific Issues and Emerging Challenges for Radiological Protection - NEA N ${ }^{\circ} 6167$, Report of the Expert Group on the Implications of Radiological Protection Science, OECD, Paris.

NEA (2008) A Stakeholder Dialogue on the implications of the ICRP Recommandations - NEA $\mathrm{N}^{\circ} 6169$, Summary of the Three NEA/ICRP Conferences, OECD, Paris.

Preston D. (2008) Circulatory diseases, Workshop on Science and values in radiological protection, Helsinki, Finland.

Sievert (1956) Studies on the gamma radiation from the body, Strahlen therapie 99, 185-195.

UNESCO (2003) Records of the General Conference, 32nd Session, Volume 1: Resolutions. United Nations Educational, Scientific and Cultural Organization, Paris, 2003, pp. 39-46.

West C.M.L., Elliott R.R., Burnet N.G. (2007) The genomic revolution and radiotherapy, Clin. Oncol. 19, 470-480. 